\title{
Discovery of novel single nucleotide polymorphisms in the bovine growth hormone receptor gene and their association with performance traits in Holstein-Friesian cattle in Ireland
}

\section{S McCabe ${ }^{1}$, S M Waters ${ }^{1}$, D J Howard ${ }^{2}$, L Giblin $^{3}$, D A Magee ${ }^{4}$, D E MacHugh ${ }^{5}$, D P Berry ${ }^{6}$}

${ }^{1}$ Teagasc, Animal Bioscience Department, Grange, Dunsany, Co. Meath, Ireland, ${ }^{2}$ Teagasc, Animal Production Research Centre, Mellows Campus, Athenry, Co. Galway, Ireland, ${ }^{3}$ Teagasc, Moorepark Food Research Centre, Fermoy, Co. Cork, Ireland, ${ }^{4}$ Animal Genomics Laboratory, UCD School of Agriculture, Food Science and Veterinary Medicine, UCD College of Life Sciences, University College Dublin, Dublin, Ireland, ${ }^{5}$ Conway Institute of Biomolecular and Biomedical Research, University College Dublin, Dublin, Ireland, ${ }^{6}$ Teagasc, Moorepark Dairy Production Research Centre, Fermoy, Co. Cork, Ireland

Email: matthew.mccabe@teagasc.ie

Introduction Growth hormone $(\mathrm{GH})$, also known as somatotrphin, is a key molecule in many of the physiological processes that are related to cattle performance traits such as milk production (Etherton and Bauman, 1998). The actions of $\mathrm{GH}$ are mediated by binding to the GH receptor (GHR). Variation in the bovine GHR gene sequence has been shown to be associated with a number of performance traits in cattle. For example, the non-synonymous single nucleotide polymorphism (SNP) F279Y in exon 8 which codes for the transmembrane domain of GHR is widely reported to be associated with milk yield and composition (Blott et al. 2003). However, there is little information on the associations of bovine GHR SNPs with performance traits in the Irish herd. The present study aimed to discover new SNPs in the bovine $G H R$ gene and quantify the associations of these, alongside previously published bovine GHR SNPs, with performance traits in Holstein-Friesian cattle in Ireland.

Materials and methods For SNP discovery, $\sim 1 \mathrm{~kb}$ of sequence, surrounding bovine SNPs reported in the Btau 4.0 build of the bovine genome ENSEMBL database, was amplified by polymerase chain reaction (PCR) of genomic DNA extracted from unique blood samples from 22 cattle of 5 different breeds ( 4 Simmenthal, 4 Angus x Holstein, 4 Belgian Blue x Holstein, 6 Holstein and 4 Charolais). PCR amplicons were sequenced bi-directionally using the Sanger method. To identify novel SNPs, 22 forward and reverse sequences for 19 amplified $\sim 1 \mathrm{~kb}$ bovine GHR gene regions were aligned using ClustalW version 2.0. Chromatograms were analysed using Chromas Lite to confirm identity of the SNPs and determine whether they were homozygous or heterozygous. Genotyping for 32 putative novel bovine GHR SNPs and 7 previously published bovine GHR SNPs was carried out on genomic DNA from 848 Holstein-Friesian sires using the with the iPLEX Gold assay (Sequenom, San Diego, CA, USA). Predicted transmitting abilities (PTAs) and their associated reliabilities for traits that were evaluated by the Irish Cattle Breeding Federation in the January 2009 domestic genetic evaluations, were available for inclusion in the analysis. PTAs were de-regressed and parental contribution to the reliability of each PTA was removed. Only sires with a reliability, less parental contribution, of $>60 \%$ were retained for inclusion in the association analysis. The association between each SNP and performance trait was quantified using weighted mixed models in ASREML with individual included as a random effect, and average expected relationships among individuals accounted for through the numerator relationship matrix. Year of birth (divided into 5 yearly intervals) and percent Holstein of the individual sire were included as fixed effects in the model. The dependent variable was de-regressed PTA, weighted by respective reliability less the parental contribution. Genotype was included in the analysis as a continuous variable coded as the number of copies of a given allele. A multiple regression mixed model (MRMM) was developed by backward elimination of the non-significant $(\mathrm{P}>0.05)$ segregating SNPs.

Results In total, 32 putative novel SNPs (30 transitions and 2 transversions, all biallelic), spanning the majority of the $\sim 300$ $\mathrm{kb}$ bovine $G H R$ gene were discovered by resequencing $\sim 19 \mathrm{~kb}$ of the gene in 22 animals of different breeds. Following genotyping of 848 Holstein-Friesians for the 32 putative novel SNPs and 7 previously published SNPs, 25 putative novel SNPs and one previously published SNP were eliminated because they were either monomorphic, had a minor allele frequency (MAF) of $<2 \%$, had abnormally high heterozygosity ( $>99.9 \%$ ), or were in complete linkage disequilibrium (LD) with another SNP. After MRMM analysis of the 13 remaining segregating SNPs (MAF 4\% - 48\%), 11 SNPs (including 7 novel SNPs) remained associated with at least one of 24 performance traits including traits for milk, growth, size and survival. Of particular interest was a novel SNP (ss159831013) in the GHR 5' non-coding region (238 kb away from F279Y) which was not in linkage disequilibrium (LD) with F279Y and remained associated with milk yield after MRMM analysis. This suggests that the ss159831013-milk yield association is independent of the $F 279 Y$-milk yield association. In addition, following MRMM analysis, there were associations of 5 novel and 5 previously published bovine GHR SNPS with 11 growth and size traits. ss159831013 and a previously published SNP (AF126288-149) in the 5' non-coding region showed associations with progeny carcass weight and angularity that both remained following MRMM analysis. Four previously published SNPs in the coding region (1 in exon 8 and 3 in exon 10) remained associated with 9 size and growth traits (1 SNP per trait) after MRMM analysis. One of these (H545) is a synonymous SNP in the bovine GHR exon 10 and was associated with 7 size and growth traits. Association of H545 with size and growth traits has not been reported previously.

Conclusions Newly discovered SNPs in the bovine GHR gene show associations with performance traits in HolsteinFriesian cattle in Ireland. These associations are independent of previously published bovine GHR SNPs and, therefore, may be of use as novel molecular breeding markers.

Acknowledgements The authors acknowledge the Irish Cattle Breeding Federation, the Irish Dairy Research Trust and the Research Stimulus Fund

\section{References}

Blott, S., Kim, J., Moisio, S., Schmidt-Kuntzel, A., Cornet, A., Berzi, P. et al., 2003. Genetics 163, 253-266.

Etherton, T.D. and Bauman, D.E. 1998. Physiological Reviews 78, 745-761. 